\title{
An Arbitrary Oriented Crack in the Box Shell
}

\section{V.I. Migdalski and V.V. Reut}

Stresses are considered in the box shell formed by two semi-infinite plates joined at right angles. The plates are similar but their thicknesses are different. The crack is an arbitrarily oriented and goes to the shell's tip. The edges of the crack are loaded in a plane of box shell's plate. It has been known that numerical methods of solving of stress state problems in the box shell weakened by a crack coming out on the tip possess bad convergence through the necessity of taking into account real singularities near the crack. The problem is solved on the assumption that the plates have small thickness with respect to the length of crack and this makes possible the consideration of problem in an asymptotical formulation, (see Popov, Reut [4]).

The initial problem is reduced to the combined state of plane and bending stress of an imaginary plate with intersecting defects whose roles are performed by the crack and the tip of box.

Using the generalized integral transforms method this problem is reduced to the one dimensional discontinuous boundary value problem in the Mellin transforms. Its solution is constructed using Green's functions. The boundary conditions on the edge of the box are satisfied exactly. After the inversion of the Mellin transforms the original problem is reduced to the system of two singular integral equation over a finite interval with the kernel of the Mellin convolution type with respect to the displacement jumps of crack's edges.

An exact solution has been obtained is quadratures by reducing the original problem to the Riemannian problem for a vector. The solution asymptotic near the point of intersecting of crack and the box's shell is investigated. Numerical results for the stress intensity factors in the apex of crack depending on the angle formed by the crack and the tip of box are given.

Under the defect we mean the line while crossing which the stresses and displacement of box have discontinuities. The advantages of this approach are on the one hand is the diminution of number of the solving equations and the quantity of boundary conditions to be fulfilled,on the other hand the solving methods for such problem are well worked out, (see Popov, Reut [3]). 
In the case of load which is symmetric relatively to crack the mathematical formulation of this problem is given by equations of the plane elasticity theory together with the conditions on the box's tip:

$$
\begin{aligned}
\langle v\rangle=0,\left\langle\tau_{r \theta}\right\rangle & =\left.K_{\theta} \tau_{r \theta}\right|_{\theta+0}, \sigma_{\theta}(r, \theta+0)=\sigma_{\theta}(r, \theta-0)=0 \\
\theta & =0, \theta=\pi, 0<r<\infty
\end{aligned}
$$

and conditions on the crack

$$
\begin{aligned}
\sigma_{\theta} & =f_{1}(r), \tau_{r \theta}=f_{2}(r) \\
\theta & =\alpha, 0<r<1
\end{aligned}
$$

Here $\langle A\rangle=A(\theta-0)-A(\theta+0), \sigma_{\theta}, \sigma_{r}, \tau_{r \theta}$ are the normal and tangential stresses, $\mathrm{u}, \mathrm{v}$ are the radial and tangential displacements respectively, $\theta>0$ corresponds to the plate with a crack, $\alpha$ is the angle between the crack and the tip of box.

$$
K_{0}=1-h_{1} / h_{2} ; K_{\pi}=1-h_{2} / h_{1}
$$

where $h_{1}, h_{2}$ are thicknesses of shell's plates.

Let us denote

$$
\begin{gathered}
\left.\langle v(r, \theta)\rangle\right|_{\theta=\alpha}=\chi(r), \\
\left.\langle u(r, \theta)\rangle\right|_{\theta=\alpha}=\mu(r), \\
0<r<\infty
\end{gathered}
$$

where $\chi(r) \equiv 0, \mu(r) \equiv 0$ when $r>1$.

Using the generalized integral transforms method (see Popov [1]) and introducing into consideration the Mellin transformations

$$
\left[\sigma_{\theta p}, \sigma_{r p}, u_{p}, v_{p}, \tau_{r \theta p}\right]=M\left[\sigma_{\theta}, \sigma_{r}, u, v r^{-1}, \tau_{r \theta} r^{-1}\right]
$$

where

$$
M[A(r)]=\int_{0}^{\infty} A(r) r^{p} d r(p \epsilon \Lambda)
$$

and $\Lambda$ is a contour lying in the strip $\{-1<\operatorname{Re} p<0,-\infty<\operatorname{Im} p<\infty\}$. Our problem can be reduced to the boundary-value problem

$$
\begin{aligned}
L\left(\sigma_{\theta p}\right) & =0 \\
\left.\sigma_{\theta p}\right|_{\theta=0, \theta=\alpha} & =0
\end{aligned}
$$

Here

$$
L \equiv \partial^{4} / \partial \theta^{4}+\left[(p+1)^{2}+(p-1)^{2}\right] \partial^{2} / \partial \theta^{2}+(p+1)^{2}(p-1)^{2}
$$

